\title{
Analysis of metabolism between high intensity physical exercise and body mass index
}

\author{
Haruka Suzuki ${ }^{1}$, Mizuki Nakajima ${ }^{1}$, Takeshi Sato ${ }^{1}$, Yousuke Takeishi ${ }^{2}$ \\ ${ }^{1}$ Laboratory of Ergonomics, Jissen Women's University, Tokyo, Japan \\ ${ }^{2}$ Kyushu Institute of Information Science, Fukuoka, Japan \\ 1517054s@jissen.ac.jp
}

\begin{abstract}
Recently new exercise prescription on International Space Station, approach to develop a higher intensity, lower duration exercise program for astronauts. There were not enough data between obese (BMI $>25)$ and high intensity physical training for health checking diagnosis The purpose of this study was to investigate of the blood in the obsess students with high intensity training performed as Sumo, Japanese-style wrestling.

Twenty five top level male Sumo wrestling player as volunteer who belong to college Sumo association in Japan and compare eight volunteer students without fitness habits participated in this study. It was performed examination of the blood below: cholesterol, total protein and glycoalbumin.

It was clearly significant difference in BMI between Sumo group and normal student. However there were normal limits in cholesterol, total protein and glycoalbumin for all subjects. There was no significant difference between Sumo group and students without fitness habits in blood test.
\end{abstract}

The major finding was that high intensity physical exercise was to maintain at normal limits by blood test in young obsess. Overall physical activity is a highly effective prescription for the prevention and management of health conditions.

Keywords: Sumo, Body Mass Index, blood test

\section{Introduction}

It was well known obesity is a global health issue. Since 2000, according to the World Health Organization (WHO), the prevalence of obesity is increasing rapidly in many countries. Obesity is related to several chronic diseases, hypertension, adverse lipid concentrations, and type 2diabetes. Especially, almost 41 million women and more than 37 million men aged 20 and over were obese in United State [1,2]. WHO reported that the obesity population of the world has increased rapidly from 1975 to 40 years, and the obesity population reached 641 million people (266 million men, 375 million women). The body mass index [BMI $=$ body weight $/$ height $\left.^{2}(\mathrm{~kg} / \mathrm{m} 2)\right]$ is the most widely used measurement of obesity at the individual and population levels. BMI does not measure the amount of body fat, and therefore, is not the best way to assess obesity, because obesity is an excess of body fat, not an excess of body weight. Japan Society for the Study of Obesity (JASSO) criterion for obesity is a BMI $>=25 \mathrm{~kg} / \mathrm{m}^{2}$, based on the relationship between BMI and morbidity and mortality for a Japanese sample. However, the WHO criterion for obesity is a BMI $>=30 \mathrm{~kg} / \mathrm{m}^{2}$.

Assessing obesity for well-trained athlete by using BMI is that it does not consider the difference in the body compositions of athlete and non-athlete subjects. Highly trained athletes usually have a larger muscle mass. Athletes can be misclassified as overweight or obese category when the BMI value for JASSO. Especially, the Sumo wrestler with high competition ability are the most remarkable example of this.

Physical exercise is particularly important as one of the habits with most influence in the control of obesity [2]. It was reported that the body rapidly became maladaptive to insufficient physical activity and continues, both the total number and the quality of life were greatly decreased. There was definitive evidence that physical inactivity was one of the important causes of most chronic diseases. In addition, chronic diseases were not necessarily the inevitable consequences of life, since physical activity predominantly prevented or slowly in chronic diseases [3]. Physical activity for Sumo training is well known beyond high intensity training as both resistance and aerobic exercise. In addition, it is shortly exercise period compare than other sports, baseball and soccer. The purpose of this study was to investigate of the metabolic function in the obesity students with high intensity training performed as Sumo, Japanese-style wrestling. Finally, our objective was to discuss a health condition nevertheless their extremely large bodies.

\section{Methods}

It was performed to simply calculate BMI as the ratio between weight $(\mathrm{kg})$ and height $\left(\mathrm{m}^{2}\right)$. It was participated twenty-five top performance male Sumo wrestling player who belong to university association of Sumo in Japan (mean \pm SD; age: $21.04 \pm 0.98$ years old; height $174.0 \pm 0.06 \mathrm{~cm}$; weight $116.52 \pm 24.28 \mathrm{~kg} ; \quad$ BMI $38.31 \pm 7.45 \mathrm{~kg} / \mathrm{m}^{2}$ ) and compared eight volunteer 
students without fitness habits (mean $\pm \mathrm{SD}$; age: $21.50 \pm 2.27$ years old; height $162.0 \pm 0.09 \mathrm{~cm}$; weight $57.03 \pm 9.15 \mathrm{~kg}$; BMI $21.44 \pm 1.25 \mathrm{~kg} / \mathrm{m}^{2)}$ as control group participated in this study. Sumo wrestling students meant a student who has been an average BMI value of 25 or more and has carried out 2 hours / day short time high intensity exercise least 5 times per week. The control group meant a general college student who did not have exercise habits.

It was performed typical blood test items below: ALB, Albumin/Globulin ratio, Glycoalbumin, cholesterol, total protein. Experimental data were expressed as mean values with standard deviation. BMI data of statistical analysis was used two-way analysis of variance (ANOVA). Statistical significance was considered at $\mathrm{p}<0.05$ by using SAS.

\section{Results}

There was a significant difference in the BMI value between the Sumo wrestling and control groups (Figure $1)$.

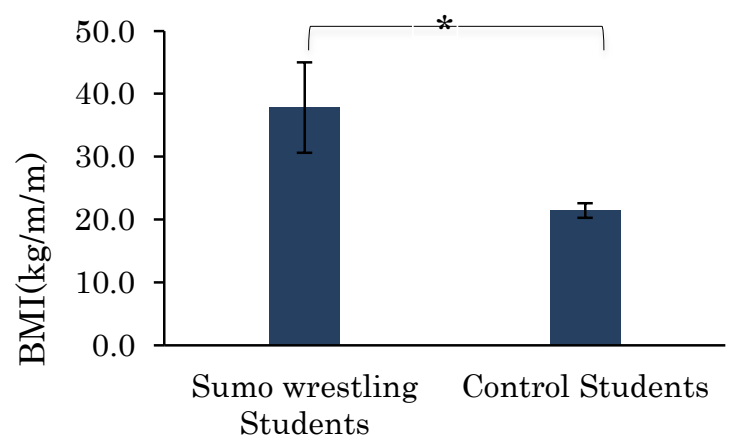

Fig.1 Comparison of BMI Sumo wrestling and control student group *: $\mathrm{p}<0.05$

As a result of the multiple comparisons, it was not significant difference that Total Protein and Albumin to globulin ratio and cholesterol experiment between two groups (Figures 2, 3).

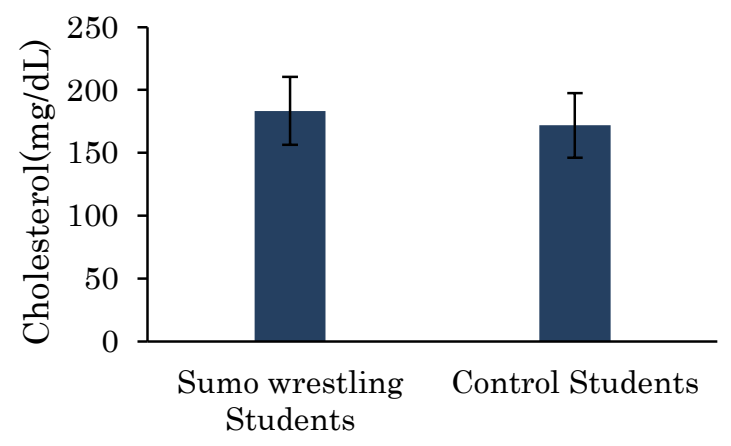

Fig2. Comparison of Cholesterol Sumo wrestling and control student group

There were reference values in cholesterol (Sumo wrestling: $181.64 \pm 28.49 \mathrm{mg} / \mathrm{dl}$, control student: $176.57 \pm 25.87 \mathrm{mg} / \mathrm{dl}$ ), total protein (Sumo wrestling: $7.48 \pm 0.38 \mathrm{~g} / \mathrm{dl}$, control student: $7.53 \pm 0.35 \mathrm{~g} / \mathrm{dl}$ ),
Albumin to globulin ratio (Sumo wrestling: $1.67 \pm 0.20$, control student: $1.78 \pm 0.19$ ), Alubmin (Sumo wrestling: $4.67 \pm 0.32 \mathrm{mg} / \mathrm{dl}$, control student: $4.78 \pm 0.25 \mathrm{mg} / \mathrm{dl})$ for all subjects. There was no significant difference between Sumo wrestling and control student.

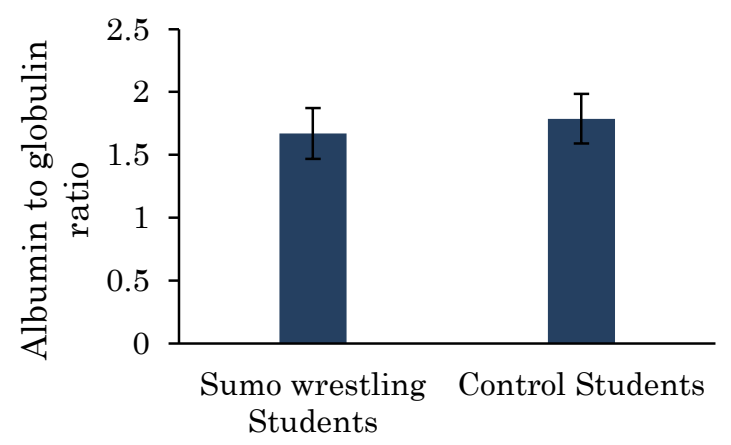

Fig.3 Comparison of Albumin to globulin ratio Sumo wrestling and control student group

\section{Discussion}

This study was to investigate the effect of high intensity and short time exercise in looking like obesity. Body Mass Index (BMI) of any group of were difference. It was reported that Total Protein and Albumin to globulin ratio and cholesterol were not significant difference. This result was shown that it was unlikely to be abnormal in biochemical indices for obese people with exercise habits.

These results supported that there were no significant difference in blood biochemical indices between healthy volunteers and sumo wrestlers (BMI> 25) (Figures 1, 2, 3 ). Increasing physical activity, then sustaining these life style changes can reduce risk of diabetes. Moreover, these results showed that repeated exercise training can overcome a distinct subset of the changes in the distal gut and fecal microbiota caused by high-fat dietinduced obesity, independently of changes in body mass or fat mass.

It was considered that body composition data was necessary to evaluate the validity of conventional BMI for assessing obesity in Sumo wrestlers by comparing them with the \%fat, \% lean body mass, to describe the unique body composition of Sumo wrestlers.

\section{References}

[1] Ahmadi M, Moosazadeh M, Vardanjani HM, DehghanA. Prevalence of obesity and overweight and their related factors among the adults of Mazandaran Province, Iran, in 2010. Electron physician, 6(4):955961,2014

[2] Kumah DB, Akuffo KO, Abaka-Cann JE, Affram DE, Osae EA. Prevalence of Overweight and Obesity among Students in the Kumasi Metropolis. J Nutr Metab, doi: 10.1155/2015/613207, 2015

[3] Frank W. Booth, Christian K. Roberts, and Matthew J. Laye. Lack of exercise is a major cause of chronic diseases.Compr Physiol,2(2): 1143-1211, 2012 